\title{
Theoretical and Conceptual Issues in Research Related to Health System Readiness and Response in Pandemic Outbreaks
}

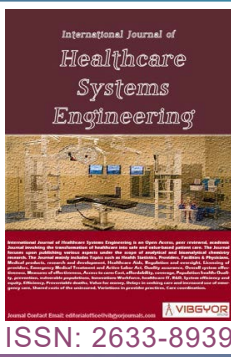

\section{William N Mkanta, PhD*, Edrisa Sanyang, PhD and Grace Lartey, PhD}

Department of Public Health, College of Health and Human Services, Western Kentucky University, United States

\begin{abstract}
An accurate assessment of the effectiveness of a health system requires integrative initiatives through its components to establish a system's response to different shocks, including health emergencies such as the COVID-19 pandemic. The focus of this study was to develop a framework for the analysis of the health system readiness and response capacity during a pandemic outbreak. A review of the conceptual and theoretical issues in developing a model for health system analysis and assessment was conducted based on the intersection and interconnectedness of the system components. An integrative modeling framework was developed by incorporating key theories derived from the system fields of preparedness, service utilization, and public health in addressing pandemic outbreaks. Three phases of an outbreak emergency have been identified and linked to integrative response effort from the three disciplines while aiming at achieving common outcome goals. The framework is designed to guide scholars, health system researchers, and policymakers in understanding and applying multidisciplinary approaches to develop effective strategies in response to health emergencies. The distribution of resources in emergencies and in the assessment of the level of preparation of the system should be among key operational processes that could benefit from adopting the context of the framework.
\end{abstract}

Keywords

System preparedness, Public health emergencies, Modeling frameworks

\section{Introduction}

The COVID-19 pandemic has brought a new dimension of global concern about the effectiveness of health systems in addressing health crisis and disease outbreaks. Following the emergence of the disease toward the end of 2019, the experience of both low-income and high-income economies has revealed varying magnitudes of deficiencies in the continuum of interventional processes along the pandemic trajectory $[1,2]$.

High-income countries such as the United States and Italy have strong health systems that typically perform at high capacity in different aspects, including workforce, equipment, technology and facilities. These systems, however, were put to the ultimate test in the wake of COVID-19 outbreak-policy makers, practitioners and politicians found themselves at crossroads in many instances when public

*Corresponding author: William N Mkanta, PhD, Department of Public Health, College of Health and Human Services, Western Kentucky University, 1906 College Heights Blvd, Bowling Green, KY 42101, United States

Accepted: May 11, 2021; Published: May 13, 2021

Copyright: (C) 2021 Mkanta WN, et al. This is an open-access article distributed under the terms of the Creative Commons Attribution License, which permits unrestricted use, distribution, and reproduction in any medium, provided the original author and source are credited.

Mkanta et al. Int J Healthc Syst Eng 2021, 3:006

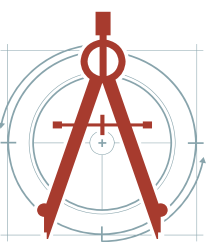


health and other measures did not seem to work adequately in controlling the outbreak [3]. The effort made by WHO in preparing scorecards on the rankings of countries based on preparedness for serious outbreaks is commended and points to the right direction on the development of sustainable preparedness at both the national and global levels. The scorecard, Global Health Security Index [4], for instance, ranked the United States first among 195 countries in health system capability, among other things, but the experience gained in the country reflected major areas of shortfalls in the response. On the other hand, for a majority of the low-income countries in the Southeastern Asia and African regions, for example, the pandemic occurred at the time when most governments were in the ongoing era of wide-spread strategies for strengthening their health systems and reducing the burden of disease. The surge of COVID-19 further exposed the structural shortfalls in these systems and the journey the governments involved should be willing to take to meet the demands of major outbreaks $[5,6]$. In essence, the lack of outbreak planning and preparedness, inadequate public health measures and poor health service interventions were seen as major components of system failure in the response to the COVID-19 pandemic.

The lack of system preparedness was evident in most regions of the world as suggested by prevalent reports of healthcare infrastructure that were not prepared to carry out the necessary tasks to establish the magnitude of the outbreak $[7,8]$. For instance, inadequate testing, poor contact tracing capabilities and delays in critical decisions were among the early signs of lapses in the systems, indicative of the lack of preparedness in both pre- and post-outbreak response. Furthermore, although many countries were successful in soliciting the services of retired and volunteer medical practitioners, or in some cases, military personnel in combating the outbreak, this measure was largely taken as a post-outbreak effort. It is further evidence of poor planning, since such effort would have added a significant value to the fight against the pandemic, had it been part of the pre-outbreak strategy.

The varying degrees of the impact of COVID-19 reported from different countries could be attributed to the unique public health issues faced in each nation. At the core of public health measures, how- ever, success in fighting the pandemic would be in the control of the spread of the coronavirus and reduction in the number of hospitalizations and deaths. Any negative outcomes seen in these areas might reflect inefficiencies ranging from the individual to systemic levels, where public health measures might be unknown, neglected, underestimated or even politicized to a degree that elevates the severity of the outbreak [7].

The United States offers an example of how devastating the impact of an outbreak could be if public health measures are not fully prioritized or undertaken. For instance, according to the World Health Organization (WHO), guidance on the adjustment of public health measures in the context of COVID-19 [9], the United States could be linked to different stages of failure in each of the four proposed indicators for risk assessment. The indicators, including epidemiological factors, health care capacity, public health surveillance capacity, and availability of pharmaceutical interventions [10], depicted patterns of suboptimal performance across communities, states and the overall system.

Although service utilization should occur in all phases of the pandemic, adequate use of services that reflect system preparedness and meets public health needs of the community, has the potential to promote positive outcomes. As the pandemic progresses, careful identification of the cases needing specific types and amounts of medical services is key to the optimal allocation and management of resources [11]. Based on the nature of the pandemic, this process should prioritize service availability according to its known or learned vulnerabilities primarily, the system should maintain ample distribution of skills and resources to address pandemic outbreaks.

The different types of system deficiencies identified in the course of the COVID-19 outbreak contributed to the severity of the pandemic in many regions of the world. In many instances, the deficiencies have caused potentially preventable outcomes such as hospitalizations and deaths $[12,13]$. At the writing of this article, the full magnitude of these outcomes was yet to be established since the pandemic was still an active threat in most parts of the world.

Interdisciplinary scholarly effort can be instrumental in generating information for improvements of the current and future system approaches in pol- 
icies and practices related to pandemic outbreaks. The aim of this study is to review methodological and conceptual issues in preparedness, public health and health services research and generate an integrative framework for research pertaining to the assessment of system capability in the response and management of pandemic outbreaks [11].

\section{Conceptual Issues}

In order to improve preparedness for infectious diseases, a clear understanding of what needs to be in place is essential. System capabilities need to be contextualized at local, regional, organizational, leadership, as well as system levels. Capability of the different levels of response means the ability to effectively identify hazards, characterize the hazard, and develop a response plan to control them in case of an emergency and ensuring equity in diverse populations with respect to prevention and utilization. In addition, capability is individual, leadership, organizational, and system's capacity to effectively identify, characterize hazards pre, during and post pandemic. By implications, this includes: 1) Preparedness; 2) Healthcare utilization; and 3) Public health and emergency response $[14,15]$.

\section{Preparedness}

Generally, hazard identification and assessment are intended to recognize and then characterize potential threat. This capability goes further to monitor the impact of the threat on the population. Hazard identification and assessment for potential infectious disease emergency depends on laboratory and surveillance capacities, including contact tracing and quarantine of infected individuals, and overall related legal arrangements before emergency event. This process is essential to support policy development, implementation, designing prevention strategies, and developing treatment protocols.

Identifying hazards that are a threat to public health, from within or outside the national borders, requires decisions about on potential effects on individual countries or regional economic blocs. Effects can be assessed in terms of impact on public health (morbidity, mortality, and disability outcomes), disruption of public health services, and overall impact on the economy. Furthermore, in infectious disease preparedness, understanding the pathogen, pathogenesis in the human host, and the environmental media are essential first steps to relate to eminence of a pandemic.

In characterizing risk of an infectious disease, it is essential to identify the pathogen and its epidemiologic characteristics including modes of transmission, risk of infection, virulence, available control strategies, and national or regional public health infrastructure. It is also essential to consider challenges posed by travelers especially in porous borders, asymptomatic patients, healthcare workers and emergency responder systems. To be effective, national disease control agencies need to provide risk results to policy makers, regional economic blocs, and multi-lateral agencies. Variables in the databases of each of these levels should be validated at all levels, otherwise characterizing the hazards will result in underestimating or overestimating the threats to the hazards. Additionally, it is important to identify appropriate personal protective equipment (PPE) or personal protective devices (PPD) for healthcare settings and emergency response, and to assure sufficient capacity to provide timely training and dissemination of information to health professionals at all levels of the system.

\section{Healthcare utilization}

Service utilization experiences drawn from the COVID-19 pandemic as well as findings from previous investigations that examined the level of preparedness of the health system in terms of the critical structural capacity in managing the demand for health services, strongly suggest further assessment is necessary to learn about the need and utilization of services in times of pandemic outbreaks. Although previous studies have attempted to demonstrate the level of resource availability $[16,17]$ they do not account for the dynamics of the need generated in the progression of an outbreak or how different components of the health system should feed each other in case of a pandemic emergency. For instance, the established system capacity (e.g., the number of ICU beds, etc.) should not be regarded as the only determinant for need for health services during a pandemic outbreak. Instead, need should be assessed as a multidimensional construct because of the limited resources and nature of the pandemic outbreaks.

Healthcare needs occurring during pandemic outbreaks are diverse and have to be addressed with responsively planned public health efforts to mitigate the effects of the pandemic. Because of 
the nature of outbreaks, characterized with rapid widespread of cases over time and geographical space, any research on service utilization should consider the diversity of the needs, unpredictability of pandemics and availability of resources in both pre-pandemic, pandemic and post-outbreak phases. In different ways, factors of utilization and phases of the pandemic outbreak introduce important conceptual issues that would play a critical role in research related to service utilization occurring during outbreaks. Even though researchers may decide to focus on specific aspects of utilization, or on utilization occurring in one of the phases of an outbreak to suit their own purposes, it is imperative that there is a body of research that purports to demonstrate the relevance of service utilization in the continuum of the pandemic outbreak. This type of research would be key in the development of resource utilization plans at different levels as well as in assessing system readiness and performance. Furthermore, it will feed the system with the current knowledge about services, effectiveness of services, and need for services related to pandemic outbreaks.

The need for health care is defined as the amount of health care that the experts believe a person should have to remain as healthy as possible, based on current medical knowledge [18]. Although this definition serves to describe the basis and anticipated outcome of service utilization, it may not be adequate to express need and ultimately utilization, i.e., the actual use of services [19], when related to outbreaks. Several conceptual issues need to be considered to develop a description that classifies need and utilization based on the characteristics of an outbreak. Since it has been demonstrated that utilization could be assessed based on the type of service, purpose of service and unit of analysis [19], it is reasonable to attempt to describe and measure both need and utilization in the context of pandemic outbreaks to generate the right type information for preparedness and intervention.

For example, different subpopulations might manifest vulnerabilities corresponding to the surge of the pandemic to generate unique patterns of need and utilization for groups such as children, elderly, persons with underlying conditions, etc. Subsequently, the public health system should offer guidance to determine differentials in vulnerability within these groups based on relevant factors, including geographic location, race, ethnicity, and socioeconomic background. That is, in agreement to the suggestion of [20], public health principles should be incorporated in conceptualizing need and utilization of services-this approach would be instrumental in identifying a framework amenable to examining need and service utilization during major outbreaks.

In general, service utilization is determined by the level of evaluated need and health status, theoretically, the two constructs are highly correlated. However due to the unpredictability nature of outbreaks, need is diversified and utilization may occur without an established need. In this case, policy interventions should be in place as one of the active health system components for application in times of outbreaks to ensure services and resources are optimally allocated to effectively mitigate the impact of an outbreak.

Considering the complexities brought into the health system when outbreaks occur, a conceptual model for service utilization combining elements of both systems approach and behavioral model of health service utilization should be integrated in the framework for research into system response and readiness for outbreaks. In systems approach, health system is viewed as consisting of interrelated components that interact with one another and with the population they serve [21,22]. Levels of care, types of practitioners, facilities and all factors of access to care are different components of the system that work together for service utilization to occur for a person or population group. Most questions about the applicability of this approach lie in determining the features of a good system and how to monitor system performance based on its components [23]. Systems approach has been used in different types of service delivery points for specific conditions, populations or settings. In the context of pandemic outbreaks, the model should include policy, preparedness and public health feedback as key system components for determining need and use of the services.

According to the behavioral model of health services use, also referred to as the Andersen model, an individual's use of health services is considered to be a function of three characteristics, namely predisposing factors, enabling factors and need factors $[19,24,25]$. It is a multilevel model which can be applied in different settings to assess indi- 
vidual and population use of services; additionally, contextual determinants of service use can also be incorporated in the model to study patterns of utilization in different populations of interest [26]. Need factors at the contextual level are of particular interest in relation to the current study. A distinction is made between environmental need characteristics, e.g., those factors reflecting the health-related conditions of the environment, including death rates due to different environmental causes, and population health characteristics, e.g., indices for overall measures of community health, including epidemiological indicators of mortality, morbidity, and disability. When applied to utilization in outbreak response, the three characteristics should be essential in the modifications needed to demonstrate how the model could be extended to address outbreak-specific contextual features of utilization. We would expect during the time of an outbreak that the system would be driven to promote equity in the distribution of resources when individuals and populations will exhibit utilization in close relation to the need generated by the outbreak rather than a person's or population's enabling power or resources [27].

\section{Public health}

Overall, adequately responding to a public health emergency requires effective management and a clear demonstration of coordination skills and control over the response systems.

Since pandemics affect a wider geographic area and a larger population, it becomes imperative for broader structures to be implemented to minimize the impact on communities and populations at large. The World Health Organization (WHO) recommends the "whole-of-society" preparedness approach in ensuring minimal impact on communities and lives [28]. This approach calls for collaboration between communities, public and private organizations in the development or updating of pandemic preparedness plans to reduce the impact [28]. Based on this premise, WHO developed the Readiness Framework which elaborates the interdependence of communities, public and private sectors in the management of a pandemic.

Socioeconomic factors influence individuals' risk for injuries and diseases including the rate of recovery. Economically disadvantaged individuals and countries are disproportionately impacted by diseases and injuries. For example, low socioeco- nomic status is linked to hospital admission and longer hospitalization and increased death [29]. Such disparities influence the outcome of the response to diseases, especially, infectious diseases where response time and effort are crucial. Several studies have examined factors influencing individual and populations' risk for pandemics and theories or models that can be used for further explanation.

Blumenshine, Reingold, Mockenhaupt, Braveman, Mark [30] extensively examined the possible disparities underlying an influenza pandemic on three (3) levels: The likelihood of being exposed to the influenza virus; likelihood of contracting influenza; and the likelihood of receiving timely and effective treatment after contracting influenza. These researchers concluded that limited health care infrastructure and disparities in health care were likely to affect the socially disadvantaged population disproportionately.

Coordination and communication between national or regional public health agencies, healthcare providers, and emergency responders is another important capability especially regarding medical transport, preventive and treatment protocols, and surveillance designs. For example, during the COVID-19 pandemic, countries around the world continue to receive guidelines for treatment, medical transport, and pathogen transmission prevention in the population from multilateral agencies like the World Health Organization (WHO), and sister national disease prevention and control agencies such as United States Centers for Disease Control and Prevention (CDC), European Union-CDC, and Africa-CDC.

Another capability essential for adequate response to a public health emergency is the development of communication system between the national public health systems and emergency response, and healthcare centers to ensure coordination of prevention and treatment efforts. The communication and coordination efforts can be related to such activities as prescription, medical supplies, and other logistical matters.

Similarly, capabilities that focuses on communications on cross-border threats requires information sharing and coordination at both regional and global levels. WHO performs this role during many pandemics including COVID-19. Immediately following the declaration of the COVID-19 pandemic, WHO began hosting regular, triweekly news brief- 
ings, which were available in multiple United $\mathrm{Na}$ tions languages. These briefings are a great source of information for not only member states, but the general population. At the national level, prescriptions and use of medical supplies and other logistical matters distributed by multilateral agencies requires additional capabilities to translate and tailor to specific national or local needs.

There is also a need for capabilities for real-time exchange of information, advice, and opinions between leadership or organizational experts or officials on one hand, and people who might be challenged with the threat (hazard) on the other hand. Such information and advice should be tailored to address issues related to prevention of community transmission, their survival, health, and economic or social well-being. The ultimate purpose of this group of capabilities is to ensure everyone at risk is able to make informed decisions for mitigating the effects of the threat (hazard) and to take protective and preventive actions. This ability extends to include the capability to segregate information to share by different population groups. People receive messages, process, and act on them differently, which is influenced by individual socioeconomic and cultural characteristics. These differences may result in inequalities within population groups and how they perceive risks and acquire knowledge during crisis, potentially affecting compliance with recommended behaviors. It should also include the capability of the system to use the most appropriate content and trusted channels of communication across population groups and to identify strategies to overcome linguistic and other cultural barriers when needed.

Communicating actual risk of public health hazards requires clear information on actual risk, uncertainty, and populations' risk perceptions in a timely manner. There is always a concern in an emergency to respond to public concerns quickly, even before all facts are known. Information should be shared without major distortion of the content. Communicating too quickly can subsequently create confusion in the population if the original message must be adapted as the facts develop. Trust on national leadership has been associated with compliance with recommended behaviors. There is a need to identify sources of information the public can trust to educate the public on roles and responsibilities of different agencies involved in the emer- gency response.

\section{The Theoretical Framework}

The varied conceptual issues arising in the assessment of health system readiness in health emergencies are considered in the stages involved in developing a modeling framework for analysis. Each of the three system components included in the framework, as discussed below, provides and justifies the necessary building blocks in this process. This reflects the importance of integration of roles in these components for appropriate system performance and assessment.

\section{Preparedness}

Hazard identification and risk assessment are the basis for a critical and successful pandemic preparedness. Numerous studies and models designed to predict hazards are getting more precise in determining when they will occur, the likely severity, and consequences on the population health and wellbeing including effects on the environment and the economy. These scientific capabilities exist in multiple countries around the world. What is however, not given an equal priority is the search for new hazards or risks during the pandemic. It is evident that as a pandemic progresses, individuals and families adjust their behaviors or manipulate the environment with the view to minimizing outbreak. The capabilities to implement such changes are different between individuals due to the differences in personal, socioeconomic, and environmental factors. Research has shown that environmental factors contribute a lot to virus behaviors, persistence, and infectibility. On the other hand, the individual's ability to manipulate the environment is dictated by a myriad of factors including purchasing power. For example, the lower a person's socioeconomic status, the more limited their resources and ability to access essential personal hygiene facilities, avoiding overcrowding or creating social distance, or even having underlying conditions such as heart disease, respiratory disease, and diabetes all of which increase the mortality risk of COVID-19 [31-33]. Individuals and families living in poverty have less control over their environment and few to no alternatives to substandard housing, transportation, childcare among other essential needs. These effects are exacerbated for people of color who are subjected to the consequences of discrimination and segregation in housing, on top of affordability challenges. Since these differences 
exist across all organizational levels, from neighborhoods, to counties, states or nations, preparedness research should factor such diversities. To be more effective, it is essential for the preparedness assessment, which includes hazard identification, risk assessment and characterization to be done throughout the continuum of the pandemic [34]. That is the most reliable approach which can provide current knowledge about the risks that emerge from the pandemic.

Generally, a population-based hazard identification and risk assessment is a systematic approach that is intended to identify and then characterize potential threat that may affect a specific population $[35,36]$. This capacity goes further to monitor the impact of the threat on the population. Hazard identification and assessment capability for a potential infectious disease emergency depends on laboratory and surveillance capacities, including contact tracing and quarantine of infected individuals, and overall related legal arrangements before an emergency event. Several countries used lessons learnt from the SARS and MERCs pandemics and developed within system strategies that were used during COVID-19 response. For example, for their COVID-19 response strategy, Taiwan used nurses as contract tracers [37].

Furthermore, it is important to note that the science and art of preparedness is a continuous cycle of planning, organizing, training, equipping, exercising, evaluating, and taking corrective action in an effort to ensure effective coordination during incident response. These levels of planning must be done within the context of different populations and sub-populations, at the same time in consideration for diversity with respect to location, race, ethnicity, and socioeconomic status. It is therefore important to incorporate public health principles in not only identifying hazards and characterizing risks, but also identifying response priorities. In general, therefore, evidence-based preparedness models should be determined by needs and vulnerability levels, the higher the population and sub-population needs, the higher the vulnerability levels, therefore the higher priority for intervention. However, characterizing the public health risk levels (or priorities) for different population and sub-population groups requires careful selection of uncertainty factors. Part of hazard characterization is to institutionalize policies to cater for, and judi- ciously allocate resources to meet the demands of those in dire need.

A preparedness research model should be based on identifying capacity and capability at system, organization, leadership, and individual levels; at the same time identifying hazards in different population and sub-population groups within the context of location, race, ethnicity, and socioeconomic status. The logic model does a great job of integrating these components leading to identifying measurable and specific outcome indicators.

\section{Healthcare utilization}

Healthcare needs occurring during pandemic outbreaks are diverse and have to be addressed with responsively planned public health efforts to mitigate the effects of the pandemic. Because of the nature of outbreaks, characterized with rapid widespread of cases over time and geographical space, any research on service utilization should consider the diversity of the needs, the unpredictability nature of pandemics and availability of resources in pre-outbreak, pandemic and post-outbreak phases. In different ways, factors of utilization and phases of the pandemic outbreak introduce important conceptual issues that would play a critical role in research related to service utilization occurring during outbreaks. Even though researchers may decide to focus on specific aspects of utilization, or on utilization occurring in one of the phases of an outbreak to suit their own purposes, it is imperative that there is a body of research that purports to demonstrate the relevance of service utilization in the continuum of the pandemic outbreak. This type of research would be key in the development of resource utilization plans at different levels as well as in assessing system readiness and performance. Furthermore, it will feed the system with the current knowledge about service types, effectiveness of services, and need for services related to pandemic outbreaks.

For example, different subpopulations might manifest vulnerabilities corresponding to the surge of the pandemic to generate unique patterns of need and utilization for groups such as children, elderly, persons with underlying conditions, etc. Subsequently, the public health system should offer guidance to determine differentials in vulnerability within these groups based on relevant factors, including geographic location, race, ethnicity, and socioeconomic background. That is, in agreement 
to the suggestion of [20], public health principles should be incorporated in conceptualizing need and utilization of services. This approach would be instrumental in identifying a model amenable to examining need and service utilization during major outbreaks.

\section{Public health}

In general, one's level of education, race/ethnicity, and economic resources underlie one's status in society including access to health care and other services [38]. Individuals disadvantaged by lower social economic status or education may not receive the same services as their counterparts on the other end of the spectrum. These diverse differences in populations and sub-populations, come with diverse behaviors mandating differences in approaches to address community and individual needs.

The Theory of Planned Behavior (TPB) has been used in several studies to explain diverse behaviors that individuals have control over. TPB is based on the concept that individuals' intention to engage in a behavior is influenced by their attitude, their beliefs about what others think, and their perception of the easiness or difficulty in engaging in the behavior [39]. Zheng, et al., [40] used the TPB in the study to examine the willingness to self-isolate when facing a pandemic risk. These researchers concluded that, perceived behavioral control (PBC), attitude (ATT) and subjective norm (SN) are key factors that influence self-isolation (SI). Similarly, Myers and Goodwin [41] used components of the extended TPB to study adults' intentions to vaccinate against the swine flu and found that PBC, ATT and SN were good predictors of intention to vaccinate.

The Health Belief Model (HBM) has also been used in studies to predict individuals' intention and behavior [41,42]. Developed in the 1950s, HBM was used to explain health behavior of individuals regarding disease prevention, early detection, and compliance to medication. HBM was later expanded to help predict the utilization of health services. The major constructs of the HBM are perceived susceptibility, perceived severity, perceived benefits and perceived barriers [43]. Perceived susceptibility is the likelihood or one's risk of contracting disease or illness; perceived severity is the individual's seriousness of contracting the disease or illness, perceived benefits is the effectiveness of available actions or resources in preventing the disease or illness; and perceived barriers refer to potential impediments to available resources or actions $[41,42]$.

Researchers have again used various models to predict and better understand the impact factors in global pandemic situations. These models include the Disease Transmission model (DTM) [44]; Global Epidemic and Mobility model (GLEaM) [45], Susceptible-Exposed-Infective-Recovered (SEIR) [46], Susceptible-Infectious-Recovered (SIR) [47].

While these models have been used extensively over time, most have focused on singular conditions and or factors. The global nature of a pandemic requires a more collaborative model that covers the broader nature of health issues, individual and population behaviors, their environment, and resources. The collective functioning of these areas would ensure the proper management of the ensuing health threats.

\section{The Framework for Health System Readi- ness in Pandemic Outbreaks}

The integrative framework for the analysis of health system readiness in pandemic outbreaks is an attempt to appeal to scholars and policy-makers on the applicability of consorted effort in outbreak management. Conceptual and practical issues discussed in the principles related to the three components of the health system, i.e., preparedness, service utilization and public health point to potential health system measures that could be taken to mitigate the impact of outbreaks. It is the premise of this study that although the system is composed of these components, their interconnectedness in the processes of care is not adequately utilized even when common goals are being pursued. In the proposed framework, we have identified sets of common goals that should guide the three system components in joining and streamlining efforts needed to manage the pre-pandemic, pandemic and post-pandemic phases of outbreaks as shown in Figure 1 and discussed in detail below.

\section{Preparedness}

Pandemic preparedness should be considered as a cycle of activities with the view to achieving goals indicated on the framework (B1 through $\mathrm{C5}$ ), including strategies to mitigate the severity of impact of the outbreak both in terms of population health effects and the economy. Hazard identification or 


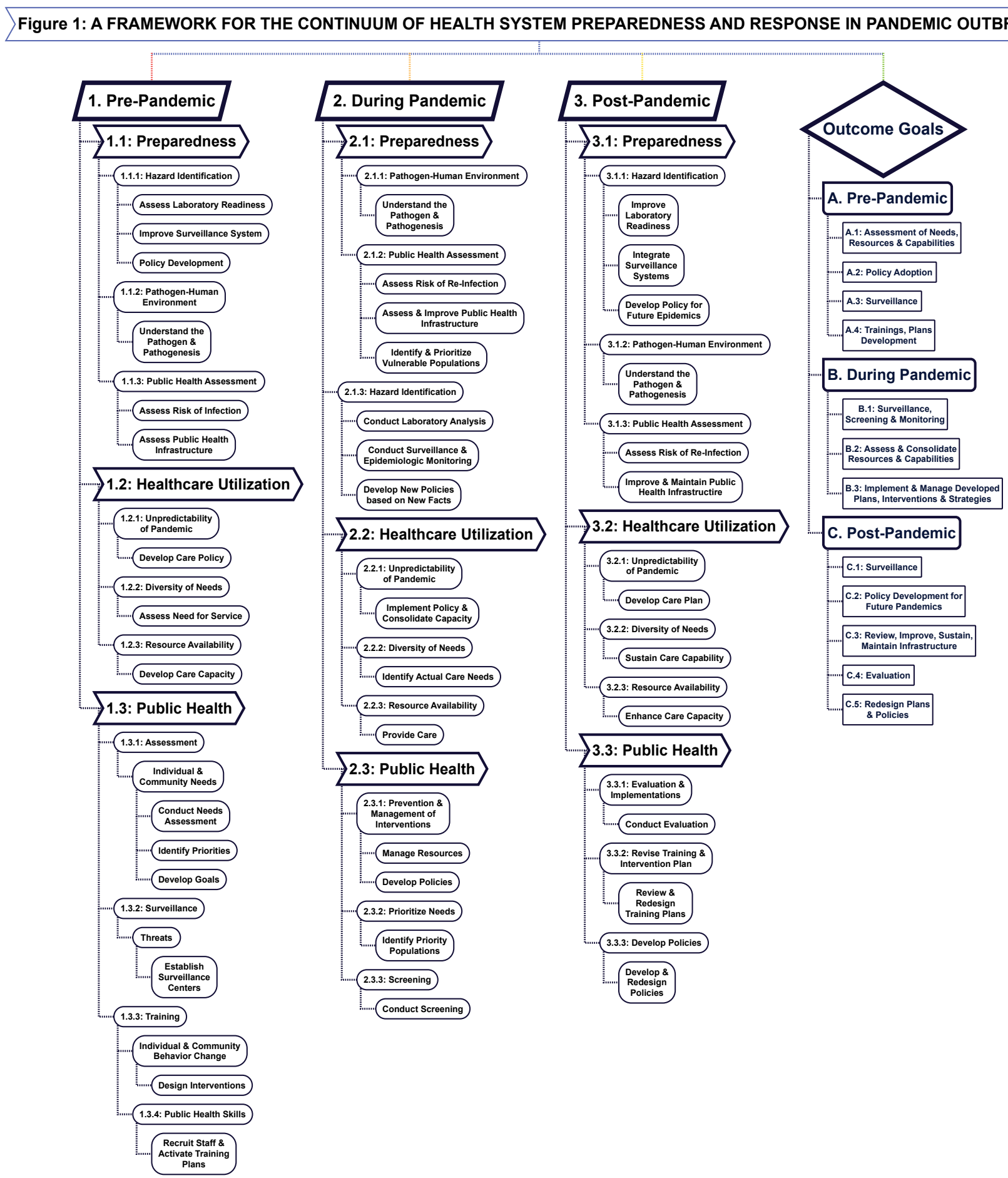

Figure 1: A Framework for the continuum of health system preparedness and response in pandemic outbreaks.

identification of potential threats to public health is the first initial step governments and healthcare systems need to identify and utilize (1.1.1). It involves such capabilities as assessing laboratory readiness, improving surveillance systems, and updating policies to adequate levels to effectively manage the outbreak in case it occurs. Additionally, understanding the pathogen-environment interactions such as identifying the type of environments which pro- mote the growth and infectivity of the pathogen and promote person to person transmission is essential (1.1.2). By extension, that implies assessing and understanding what demographic profiles of the population and sub-populations will be more susceptible to the infection (1.1.3). The public health assessment will also include assessing and understanding the capabilities of public health infrastructure and determining the preparedness level. 
During pandemic, the government or healthcare agency's capability to identify and understand pathogen behavior is important to design appropriate environmental manipulations (2.1.1). For example, it is the pathogen behavior that helps to determine if such strategies such as social distancing, coughing in the elbow, or even improving set up in clinic, home and public space environments are warranted to minimize transmission in the population. A related capability is the ability to access public health readiness for such factors as risk of infection for those who already have been infected (or the possibility of creating herd immunity) in the population (2.1.3). This capability also includes the ability to assess the potential for re-infection, identify and prioritize services for the population and sub-populations that are likely to be severely affected by the outbreak. Another critical step during pandemics is to conduct laboratory analysis of cases not just to confirm them and take appropriate steps to isolate and prevent community transmission, but also, understand the pathogen better (2.1.1). To be effective, this should go along side with active surveillance including epidemiologic monitoring. The structures and variables in the surveillance system need to be fairly consistent across countries or regional economic blocks. At a minimum, having one system for each of the WHO regions is critical. What is in fact desirable is to have a standard surveillance system across all countries.

The post-pandemic phase assessment is aimed at identifying potentials for future threats (3.1.1). The hazard identification activities in this phase involves developing strategies to improve laboratory readiness, integrating epidemiologic and surveillance systems and developing policies based on information from continuous data collection efforts in preparation for future outbreaks. As part of the hazard identification efforts, the strategies assessing capabilities to understand pathogen-environment interactions especially with respect to potential for mutation and developing of new strains of the virus are essential (3.1.2). Models can also be developed to present different scenarios in recognition for like effects on population and sub-populations (3.1.3). In addition, model predictions can be used to improve and maintain public health infrastructure.

Community capacities and capabilities have to be assessed to identify their needs, resources, threats, and opportunities. This assessment in- cludes at risk populations, personnel, infrastructure, equipment, and supplies. Available opportunities within the community in addition to barriers and threats should also be identified for resolution (A.1). Surveillance systems have to be developed to be able to track initial cases to prevent the spread among the populace (A.3). Leaders should review existing health policies and revise them or develop new ones (A.2). Finally, training and intervention plans and strategies should be reviewed and designed (A.4).

The surveillance started in the pre-pandemic phase continues in the pandemic phase. In addition, the population should be screened to identify early onset of cases for treatment. Lessons from the SARS-COV-2 indicate asymptomatic individuals spread the virus. Identified cases must be treated and monitored to ensure their quick return to normalcy (B.1).

The assessment of resources and capabilities continues during the pandemic phase. There may be the reallocation of resources to the most needed places or where more infrastructure is needed to support the current load (B.2). Developed plans, interventions and strategies during the pre-pandemic phase have to be implemented during the pandemic phase (B.3).

Continuous surveillance is essential to ensure new cases are quickly isolated and monitored to prevent new infections and spread among the community (C.1). An evaluation of the entire system is crucial at this point to assess which of the strategies worked, those that did not work and the plans that need to be revised (C.3 \& 4). This prevents unnecessary delay and a repeat of the same mistakes during the next incident. Efforts should be made to sustain the strategies, policies and plans that worked in remediating the pandemic. Finally, the redesign of plans, strategies, interventions and policies should start to ensure the system's readiness.

\section{Healthcare utilization}

In the pre-pandemic phase, due to the unpredictability of outbreaks (1.2.1) where the nature of any impending outbreak is unknown, policies, including how to obtain services and guidelines on health services provision should be developed and adopted as part of the system component. The diversity of need that could be potentially generated 
by an outbreak (1.2.2), should lead to system efforts in assessing the magnitude of need based on the projections and estimates of the need at the time of an outbreak. According to the Andersen model, need should be the main driver of utilization, which implies a broad consideration of the different dimensions of need in an outbreak should be done. Another important aspect of utilization under the pre-pandemic phase of an outbreak is resource availability (1.2.3). All actions undertaken to ensure the system as a whole has the capacity to meet the need, and at the same time prevent maldistribution of resources in the system should be taken during this phase. As these actions are taken in unison with the system components of preparedness and public health, the pre-pandemic outcome goals ( $A 1$ to $A 4$ ) should be realized and can be assessed at different levels to ensure the system is adequately prepared for pandemic outbreaks.

During the pandemic, the goals indicated on the framework (B1 to B3) have to be met for the system to be able to mitigate the impact of the outbreak in terms of both number of deaths and severity of the cases. The policies developed in pre-pandemic phase will be implemented to allow the corresponding utilization of health services to occur (2.2.1). A point of emphasis here is the consolidation of effort in the system in understanding the nature of the outbreak to ensure efficiencies are attained across the functioning components of the system- i.e., a systems approach. The actual need for care is determined by health professionals based on the assessments made on the effects of the outbreak (2.2.2). More importantly, this should be the need evaluated based on the vulnerabilities associated with the pandemic. This implies, according to the Andersen model, the system has to have the capability of using its different components to establish need of different population groups for appropriate care to take place. The diversity of needs (2.2.2), for instance, in an efficient system, should have assisted in understanding the need of the elder population that has been severely impacted by the coronavirus pandemic. Resources developed and prepared for pandemic outbreaks would be directed in all the processes of care to facilitate utilization of health services among those impacted by the outbreak (2.2.3). Care provided during this phase of the outbreak should be sensitive to the need of different groups in the population based on the assessments made under 2.2.2.
The post-pandemic phase is also an essential part of service utilization according to the integrative framework. During this period, the outbreak will be generally under control, but there will be cases as well as individuals suffering from the residual effects of the outbreak needing the attention of the system as indicated under the provisions of 2.2.3. However, service utilization occurring in this phase of the pandemic should represent the joint effort in the system components to attain post-pandemic goals $\mathrm{C} 1$ to $\mathrm{C} 5$ as a way of closing the loop in the system's effort to address the outbreak. In this manner, this phase should end with an upgrade of the system in its pre-pandemic level for any future outbreaks. Based on the systems approach and the Andersen behavioral model, actions on service utilization provisions undertaken through stages included under 3.2.1, 3.2.2, and 3.2.3 should prompt the system components to enhance enabling factors for future efficiencies at all levels of health service utilization. These measures will address any barriers thought to hinder care processes during the pandemic phase and will be key in redesigning utilization plans and policies (C5).

\section{Public health}

As part of the preparation towards a pandemic, the health services system including the community has to be assessed in terms of available resources and challenges (1.3.1). This phase will ensure the identification of priority areas to focus resources, develop goals and objectives and start the planning process. Challenges identified will also be addressed such as equipping personnel with the needed skills and infrastructure development.

The community and individual behavior and attitudinal change process must also be initiated at this time for disease prevention. This calls for the activation of infection prevention, health education, and awareness programs. To be proactive, infection surveillance system should be activated at this time (1.3.2). This will help with the prompt identification of initial cases, reduce the spread of the pandemic, manage cases promptly, and thereby not overwhelm the health care system and other resources.

Infection prevention strategies should continue during the pandemic (2.3) to protect those who have not been affected yet. Besides, infection management interventions should be fully operational at this point. Quarantine and isolation procedures 
must be employed together with treatment regimen. Screening of the population to identify those who have contracted the pathogen is essential during this period. These efforts will reduce the spread and manage those infected better. Resources needed to be successful in this regard should be made available to the communities, treatment centers and other areas responsible for handling the pandemic. Continuous assessment of resources and the entire system is needed during this phase as any shortage will cause the collapse of the system and increase the infection burden. Existing health policies must be activated, and new ones enacted at the various levels to support these efforts. Regular assessment of activated plans/interventions needs to occur for any needed changes to be made.

Post-pandemic phase (3.3) is where we utilize the framework to review what worked well, what did not, and what changes are needed. Evaluation of the entire system and process has to be completed to identify successes and mistakes made during the pre-pandemic and pandemic phases. Training and retraining of personnel and the public in prevention and management strategies, as well as review and revisions of intervention plans, policies, and assessment of resources (1.3.3). These activities will ensure the system is better prepared for prevention and management of future pandemics.

\section{Discussion}

The aim of this study was to review theoretical and conceptual issues in preparedness, public health and health services research and generate an integrative model for research pertaining to the assessment of system capability in the response and management of pandemic outbreaks.

The impact of the COVID-19 pandemic has affected individuals, communities and nations in diverse ways. In addition to the enormous global morbidity and mortality burden, economies are struggling to remain viable. Schools have been closed or running on-half capacity in most countries, thereby putting more strain on the already struggling education systems. While the novelty of the pandemic has made it difficult for containment strategies to succeed, the question falls back to the response and preparedness of communities and nations. Either the communities and nations were not prepared, or their preparations and response were inadequate to withstand the impact of this pandemic.
An improvement in community preparedness and readiness is important in the prevention and management of pandemics.

Our review identified various theories and models for healthcare utilization, preparedness and public health, however, none combined these three areas. These three system components overlap in many ways while moving towards the common goal of outbreak control. System review and assessment efforts should benefit from the integration of these components as well.

Secondly, infections have no timelines. Health system response and preparedness should start before cases begin to appear. While capabilities and resources cannot be activated until cases are recorded, surveillance, intervention, strategic plans and policies can be developed early on at the pre-pandemic phase. These will be activated as soon as the first cases appear.

Most communities and nations' strategies end at the pandemic phase. Research has shown that the post-pandemic phase in preparation is essential. The evaluation of the entire system helps in identifying the strengths and weakness of the capabilities, strategies, interventions, plans and policies for changes to be made. Since infections do not vanish overnight, continuous monitoring is required to protect the population and for systems improvement. These efforts together will improve on the preparedness and response capacity of the system.

The threat and re-emergence of infectious diseases is gradually becoming a global issue. The last few years have seen new diseases such as the H1N1 pandemic in 2009; the global response uncovered the lapses in the health services systems of many countries and communities. Although the impact of H1N1 was not the same worldwide, the World Health Organization (WHO) and countries became aware of the imminent danger posed by infectious diseases. It was essential for communities to be prepared in their response towards next disease outbreak.

With the emergence of SARS-COV-2's in late 2019 , the spread and global response prompted researchers to review the methodological and conceptual issues associated with preparedness, public health and healthcare utilization of communities. The review was conducted in three areas: Pre-pan- 
demic, pandemic and post-pandemic under the three broad areas of preparedness, public health and healthcare utilization. While these three areas seem separate, the activities under each including the broad areas are not mutually exclusive (Figure 1 ) as seen in the outcome goals.

The framework presented in this article identifies what capabilities and capacities are critical at every stage of the pandemic preparedness and response. Moreover, the interconnectedness of the system components will provide insight into identifying more goals and activities that are appropriate for individual communities, countries, regions, or entities. The framework also provides details on the capacities and capabilities to measure especially during emergency preparedness exercises, critical analysis, identification of healthcare needs and service utilizations, and other public health approaches both in terms of pandemic preparedness as well as during response and planning for further emergency events.

Focusing on common outcome measures and variables at all the three phases of the pandemic provide measurements that allows for comparisons at state, national and regional levels. It also provides an understanding of pandemic scenarios over time. One of the most important reasons for preparedness and response is to minimize population health effects and effects on the economy, but also, importantly, to learn the pitfalls and work on them in preparation for future events. The elements in the framework provide excellent guide to provide basis for the structure of any form of pandemic.

The methodological and conceptual issues in preparedness, healthcare service utilization, and public health presented in the framework can also have program planning applications. Variables in the framework can be used to develop competency-based trainings to different health and emergency response professionals, and at different phases of the pandemic. Training needs can range from laboratory capacity and capabilities, epidemiologic and disease surveillance, assessing healthcare needs and develop health utilization plans including inventory of resources and projections among others. Due to differences in population and sub-population needs at local, state, national, regional levels, public health agencies need approaches that effectively address the needs of different people, especially the excluded and the deprived individuals and families.

We expect scholars with research focus on the health system components discussed in the integrative framework in this study, as well as those interested in closely related fields, will continue to expand their research to conduct further investigations to assess the degree of system readiness prior to outbreaks and other emergencies. The integrative framework is a guide for future system researchers who should use its context to determine the nature and levels of the interconnectedness of the system components for their investigations. This approach has the potential to generate effective measures for system performance and improvements in different aspects of care beyond those related to response to health emergencies. While the framework presented in this study focused on three system components, its structure can be modified to include any number of relevant components for assessments that can be applied to different units of analysis such as organizations, leadership in health care, geographic areas, and individuals. It can also assess performance patterns established over time.

While we have attempted to demonstrate how the integrative framework could be applied in analyzing the health system, we recognize that the framework is heavily relying on the assumption that system components can be linked together for analyses. Although this is a plausible assumption, there is a possibility for arguments to emerge on the way it simplifies the linkages. This can be especially a barrier in the adoption of the framework when issues surrounding data and resource availability are considered. Whereas this may be viewed as one of the limitations of the framework, we acknowledge the thoughts presented here is also work in progress toward the contribution scholars can make in developing research ideas and collaborations that could contribute to the efficiencies sought in the health system. The possible modifications that could be made on the framework in terms of system components, phases of the outbreak, or types of health emergencies could be regarded as measures to address any limitations inherent in the application of the framework.

In conclusion, our study signifies the crucial importance of promoting collaborative effort in building an effective health system. We have developed 
a modeling framework that could guide and assist both scholars and policymakers in assessing the effectiveness of the health system in situations involving health emergencies. The toll of the COVID-19 pandemic on the U.S. health system has provided important lessons on the shortfalls that primarily pointed to the lack of cohesion in the system components in the management of the outbreak. It is our anticipation that ideas, concepts, and the framework developed in this study will receive the attention of a wide forum of investigators, public health and health management workforce, and policy makers. These are among the entities that should recognize the importance of using the modeling framework on the existing health system capacities and capabilities to develop more effective and responsive processes. Such initiatives should be a priority for projections leading to integrated strategies for the control and mitigation of outbreaks and health emergencies.

\section{References}

1. Coccolini F, Sartelli M, Kluger Y, Pikoulis E, Karamagioli E, et al. (2020) COVID-19 the showdown for mass casualty preparedness and management: The cassandra syndrome. World J Emerg Surg 15: 26.

2. Remuzzi A, Remuzzi G (2020) COVID-19 and Italy: What next? Lancet 395: 1225-1228.

3. Gaffney A, Himmelstein DU, Woolhandler S (2020) COVID-19 and US health financing: Perils and possibilities. Int J Health Serv 50: 396-407.

4. Cameron EE, Nuzzo JB, Bell JA (2019) 2019 Global health security index.

5. Bong $\mathrm{CL}$, Brasher $\mathrm{C}$, Chikumba E, McDougall R, Mellin-Olsen J, et al. (2020) The COVID-19 pandemic: Effects on low- and middle-income countries. Anesth Analg 131: 86-92.

6. McMahon DE, Peters GA, Ivers LC, Freeman EE (2020) Global resource shortages during COVID-19: Bad news for low-income countries. PLoS Negl Trop Dis 14: e0008412.

7. Benjamin GC (2020) Ensuring health equity during the COVID-19 pandemic: The role of public health infrastructure. Rev Panam Salud Publica 44: e70.

8. Villa S, Lombard A, Bozzi A, Gori A, Raviglione MC (2020) The COVID-19 pandemic preparedness or lack thereof: From China to Italy. Glob Health Med 2: 7377.

9. World Health Organization (2020) Considerations for implementing and adjusting public health and social measures in the context of COVID-19: Interim guidance.

10.World Health Organization (2020) Public health criteria to adjust public health and social measures in the context of COVID-19.

11.Nicola M, Alsafi Z, Sohrabi C, Kerwan A, Al-Jabir A, et al. (2020) The socio-economic implications of the coronavirus panademic (COVID-19): A review. Int J Surg 78: 185-193.

12.Price-Haywood EG, Burton J, Fort D, Seoane L (2020) Hospitalization and mortality among black patients and white patients with Covid-19. N Engl J Med 382: 2534-2543.

13. Rentsch CT, Kidwai-Khan F, Tate JP, Park LS, King JT, et al. (2020) COVID-19 by race and ethnicity: A national cohort study of 6 million United States veterans. medRxiv, 20099135.

14. Hutchins SS, Fiscella K, Levine RS, Ompad DC, McDonald M (2009) Protection of racial/ethnic minority populations during an influenza pandemic. Am J Public Health 99: S261-S270.

15. Qari SH, Yusuf HR, Groseclose SL, Leinhos MR, Carbone EG (2019) Public health emergency preparedness system evaluation criteria and performance metrics: A review of contributions of the CDC-funded preparedness and emergency response research centers. Disaster Med Public Health Prep 13: 626-638.

16. Anthony C, Thomas TJ, Berg BM, Burke RV, Upperman JS (2017) Factors associated with preparedness of the US healthcare system to respond to a pediatric surge during infectious disease pandemic: Is our nation prepared? Am J Disaster Med 12: 203-226.

17. Kain T, Fowler $R$ (2019) Preparing intensive care for the next pandemic influenza. Crit Care 23: 337.

18. Feldstein PJ (2012) Health care economics. Cengage Learning.

19. Andersen RM (1995) Revisiting the behavioral model and access to medical care: Does it matter? J Health Soc Behav 36: 1-10.

20.Derose KP, Gresenz CR, Ringel JS (2011) Understanding disparities in health care access--and reducing them--through a focus on public health. Health Aff (Millwood) 30: 1844-1851.

21.Andersen JG (1973) Health services utilization: Framework and review. Health Serv Res 8: 184-199.

22.Shao S, Wang M, Jin G, Zhao Y, Lu X, et al. (2018) Analysis of health service utilization of migrants in Beijing using Anderson health service utilization model. BMC Health Serv Res 18: 462. 
23.Clarkson J, Dean J, Ward J, Komashie A, Blashford $T$ (2018) A systems approach to healthcare: From thinking to practice. Future Healthc J 5: 151-155.

24.Andersen R, Bozzette S, Shapiro M, St Clair P, Morton $S$, et al. (2000) Access of vulnerable groups to antiretroviral therapy among persons in care for HIV disease in the United States. HCSUS consortium. HIV cost and services utilization study. Health Serv Res 35: 389-416.

25. Babitsch B, Gohl D, Von Lengerke T (2012) Re-revisting Andersen's behaviroal model of health services use: A systematic review of studies from 1998-2011. Psycho-social Medine, 9.

26.Andersen RM, Rice TH, Kominski GF (2011) Changing the US health care system: Key issues in health services policy and management. John Wiley \& Sons.

27.Chatterjee PK (2020) Community preparedness for COVID-19 and frontline health workers in Chhattisgarh. Indian J Public Health 64: S102-S104.

28. World Health Organization (2009) Whole-of-society pandemic readiness.

29.Bein T, Hackner K, Zou T, Schultes S, Bosch T, et al. (2012) Socioeconomic status, severity of disease and level of family members' care in adult surgical intensive care patients: The prospective ECSSTASI study. Intensive Care Med 38: 612-619.

30.Blumenshine $P$, Reingold A, Egerter S, Mockenhaupt R, Braveman P, et al. (2008) Pandemic influenza planning in the United States from a health disparities perspective. Emerg Infect Dis 14: 709-715.

31.Giannouchos TV, Sussman RA, Mier JM, Poulas K, Farsalinos K (2020) Characteristics and risk factors for COVID-19 diagnosis and adverse outcomes in Mexico: An analysis of 89,756 laboratory-confirmed COVID-19 cases. Eur Respir J 57: 2002144.

32. Kretchy IA, Asiedu-Danso M, Kretchy JP (2020) Medication management and adherence during the COVID-19 pandemic: Perspectives and experiences from low-and middle-income countries. Res Social Adm Pharm 17: 2023-2026.

33.Prabhakaran D, Perel P, Roy A, Singh K, Raspail L, et al. (2020) Management of cardiovascular disease patients with confirmed or suspected COVID-19 in limited resource settings. Glob Heart 15: 44.

34.Ario AR, Makumbi I, Bulage L, Kyazze S, Kayiwa J, et al. (2019) The logic model for Uganda's health sector preparedness for public health threats and emergencies. Glob Health Action 12: 1664103.
35.Du Y, Ding Y, Li Z, Cao G (2015) The role of hazard vulnerability assessments in disaster preparedness and prevention in China. Mil Med Res 2: 27.

36.Stoto MA, Nelson C, Savoia E, Ljungqvist I, Ciotti M (2017) A public health preparedness logic model: Assessing preparedness for cross-border threats in the European region. Health Secur 15: 473-482.

37. Huang LH, Chen CM, Chen SF, Wang HH (2020) Roles of nurses and national nurses associations in combating COVID-19: Taiwan experience. Int Nurs Rev 67: 318-322.

38. Braveman PA, Egerter SA, Cubbin C, Marchi KS (2004) An approach to studying social disparities in health care. Am J Public Health 94: 2139-2148.

39.Ajzen I (1991) The theory of planned behavior. Organizational Behavior and Human Decision 50: 179211.

40.Zheng F, Liao C, Fan QH, Chen HB, Zhao XG, et al. (2020) Clinical characteristics of children with coronavirus disease 2019 in Hubei, China. Curr Med Sci 40: $275-280$.

41. Myers LB, Goodwin R (2011) Determinants of adults' intention to vaccinate against the pandemic swine flu. BMC Public Health 11: 15.

42.Cummings KM, Jette AM, Brock BM, Haefner DP (1979) Psychosocial determinants of immunization behavior in a swine influenza campaign. Med Care 17: 639-649.

43. Rosenstock IM (1974) Historical origin of the health belief model. Health Education Monogram 2: 328335.

44.Ajelli M, Merler S, Pugliese A, Pizzo C (2010) Model predictions and evaluation of possible control strategies for the $2009 \mathrm{~A} / \mathrm{H} 1 \mathrm{~N} 1 \mathrm{v}$ influenza pandemic in Italy. Epidemiol Infect 139: 68-79.

45.Bajardi P, Poletto C, Ramasco JJ, Tizzoni M, Colizza V, et al. (2011) Human mobility networks, travel restrictions, and the global spread of 2009 H1N1 pandemic. PLoS One 6: e16591.

46. Godio A, Pace F, Vergnano A (2020) SEIR modeling of the Italian epidemic of SARS-CoV-2 using computational swarm intelligence. Int J Environ Res Public Health 17: 3535.

47. Bootsma MC, Ferguson M (2007) The effect of public health measures on the 1918 influenza pandemic in U.S. cities. Proc Natl Acad Sci USA 104: 7588-7593. 